\title{
Agarose-based spheroid culture enhanced stemness and promoted odontogenic differentiation potential of human dental follicle cells in vitro
}

\author{
Min $\mathrm{Li}^{1} \cdot$ Tiwei $\mathrm{Fu}^{2} \cdot$ Sen $\mathrm{Yang}^{3} \cdot$ Lanlan Pan ${ }^{4} \cdot$ Jing Tang ${ }^{1} \cdot$ Meng Chen ${ }^{5} \cdot$ Panpan Liang ${ }^{2} \cdot$ Zhi Gao $^{1} \cdot$ Lijuan Guo $^{1,6}$
}

Received: 19 March 2021 / Accepted: 9 May 2021 / Published online: 1 July 2021 / Editor: Tetsuji Okamoto

(C) The Society for In Vitro Biology 2021

\begin{abstract}
Human dental follicle cells (HDFCs) are an ideal cell source of stem cells for dental tissue repair and regeneration and they have great potential for regenerative medicine applications. However, the conventional monolayer culture usually reduces cell proliferation and differentiation potential due to the continuous passage during in vitro expansion. In this study, primary HDFC spheroids were generated on $1 \%$ agarose, and the HDFCs spontaneously formed cell spheroids in the agarose-coated dishes. Compared with monolayer culture, the spheroid-derived HDFCs exhibited increased proliferative ability for later passage HDFCs as analysed by Cell Counting Kit-8 (CCK-8). The transcription-quantitative polymerase chain reaction (qRT-PCR), western blot and immunofluorescence assay showed that the expression of stemness marker genes Sox2, Oct4 and Nanog was increased significantly in the HDFC spheroids. Furthermore, we found that the odontogenic differentiation capability of HDFCs was significantly improved by spheroid culture in the agarose-coated dishes. On the other hand, the osteogenic differentiation capability was weakened compared with monolayer culture. Our results suggest that spheroid formation of HDFCs in agarosecoated dishes partially restores the proliferative ability of HDFCs at later passages, enhances their stemness and improves odontogenic differentiation capability in vitro. Therefore, spheroid formation of HDFCs has great therapeutic potential for stem cell clinical therapy.
\end{abstract}

Keywords Agarose $\cdot$ Spheroid · Odontogenic differentiation $\cdot$ Human dental follicle cells

Zhi Gao

gqyidi2001@163.com

Lijuan Guo

663418587@QQ.com

1 Department of Stomatology, The Second Affiliated Hospital of Chongqing Medical University, 76 Linjiang Road, Yuzhong District, Chongqing 400010, People's Republic of China

2 Chongqing Medical University Stomatology College, Chongqing Key Laboratory for Oral Diseases and Biomedical Sciences, Chongqing Municipal Key Laboratory of Oral Biomedical Engineering of Higher Education, Chongqing 401147, People's Republic of China

3 Stomatology Centre, Suining Central Hospital, Suining 629000, People's Republic of China

4 Department of Periodontics, Stomatology Hospital of Chongqing Medical University, Chongqing 401147, People's Republic of China

5 Department of Endodontics, Stomatology Hospital of Chongqing Medical University, Chongqing 401147, People's Republic of China

6 Department of Medical Cosmetology, Suining Central Hospital, Suining 629000, People's Republic of China

\section{Introduction}

Multipotent mesenchymal stromal cells (MSCs) have been extensively explored as a stem cell source for several clinical applications, including cancer, graft-versus-host disease, neurodegenerative disease, perianal fistulas, cardiac disorders and COVID-19 (Levy, et al. 2020). A large number of MSCs require in vitro amplification because the number of cells isolated from tissue is too small for therapeutic applications (Mushahary, et al. 2018). The traditional methods for cell culture and amplification are mainly used two-dimensional culture techniques. It has been shown that conventional 2D monolayer culture influences cell behaviour, resulting in cell senescence, loss of cell proliferation, impaired multipotency and loss of differentiation potential by continuous passages during in vitro expansion (Charriere, et al. 2010, McKee and Chaudhry, 2017a). Therefore, large-scale expansion of MSCs maintaining the proliferation and stemness in vitro and identifying optimal culture conditions is essential for clinical therapeutic needs. 
In their tissue microenvironments, stem cells maintain their stemness properties by biochemical and biophysical factors collectively termed the stem cell niche, which comprises soluble growth factors, cell-cell interactions and cell-extracellular matrix interactions (Watt and Hogan, 2000, Morrison and Spradling, 2008, Discher, et al. 2009). Compared with conventional monolayer cultures, three-dimensional (3D) culture methods are a new approach for the expansion of MSCs and offer a cellular niche mimicking the in vivo environment (McKee and Chaudhry, 2017b). Several previous studies have demonstrated that MSCs cultured in 3D spheroids show enhanced cell viability, stemness, differentiation potential and antiinflammatory properties compared to monolayer cells (Bartosh, et al. 2010, Huang, et al. 2011, Cheng, et al. 2012). Furthermore, a recent study demonstrated that coculture of late passage adipose-derived stem cells (ASCs) and endothelial colony-forming cells (ECFCs) can restore native ASC function by reversing cellular senescence in vitro. When transplanted into mice with ASC/ECFC, 3D spheroids demonstrated to have pro-angiogenic capacity and promote healing ( $\mathrm{Hu}$, et al. 2020). Our previous studies have shown that the rat dental follicle stem cells (rDFSCs) exhibited spheroid growth in Matrigel 3D cultures and promoted BMP9-induced bone formation $(\mathrm{Fu}$, et al. 2019). Multicellular spheroid culture is the simplest $3 \mathrm{D}$ culture methods, allowing 3D interactions between the cells and the ECM in the absence of additional substrates (Baraniak and McDevitt, 2012). Hydrogels such as collagen (Yamada, et al. 2015), Matrigel (Miao, et al. 2014), chitosan (Fukuda, et al. 2006) and agarose (Kuwashima, et al. 1993) can be used as bio-mimicking scaffolds for MCSs to induce cell-matrix interactions in multicellular spheroids. Agarose is a natural polysaccharide polymer and particularly attractive as its excellent biocompatibility, low cost and thermo-reversible gelation behaviour can induce spheroid formation and the spheroids' stemness expression increased significantly and enhanced adipogenesis and chondrogenesis differentiation potential of human adipose-derived stem cells (Tsai, et al. 2019). Moreover, agarose as its permeability character to gas and small biomolecules can be applied to evaluate the efficiency of anticancer drugs (Tang, et al. 2016). However, it is unclear whether the stemness properties and differentiation potential of HDFCs can be maintained or improved by spheroid culture.

In the present study, we employed an agarose culture method that is easy and effective to produce spontaneous spheroid in vitro. We assessed whether the proliferative activity and stemness properties of 3D spheroid HDFCs are improved than those of 2D monolayer HDFCs. In addition, we further examined the odonto/osteogenic differentiation potential of 3D spheroid HDFCs in vitro.

\section{Materials and methods}

Isolation and culture of HDFCs Primary human HDFCs were isolated from third molars of dental follicle tissue obtained during surgical extracted wisdom teeth as previously described. The Ethics Committee approved all procedures of Chongqing Medical University, and all samples were collected with informed consents. Dental follicle tissue was cut into small pieces and vibrated for $30 \mathrm{~min}$ in an enzyme solution containing collagenase I $(1 \mathrm{mg} / \mathrm{mL})$ that degrades the extracellular matrix at $37^{\circ} \mathrm{C}$. The suspension was collected by centrifugation at $1000 \mathrm{rpm}$, and the single cells and tissue pieces transferred to $\alpha$-modified Eagle's medium ( $\alpha$-MEM, (Gibco, Grand Island, NY) supplemented with 10\% FBS (Gibco) and $1 \%$ penicillin-streptomycin (Gibco) in a humidified incubator at $37^{\circ} \mathrm{C}$ with $5 \% \mathrm{CO}_{2}$ for $72 \mathrm{~h}$. For differentiation, cells were grown to confluence and induced with differentiation medium (10 mM $\beta$-glycerophosphate, $100 \mathrm{nM}$ dexamethasone and 50 $\mu \mathrm{g} / \mathrm{mL}$ ascorbic acid).

Flow cytometric For flow cytometry analysis, HDFCs were harvested at passage 2, $10^{6}$ suspended cells were washed and stained by incubation with the following antibodies and corresponding isotype controls: FITC-conjugated mouse antihuman CD34 (BD Biosciences, San Diego, CA), FITCconjugated mouse antihuman CD45 (BD Biosciences), FITC-conjugated mouse antihuman CD90 (BD Biosciences) and FITC-conjugated mouse antihuman CD105 (BD Biosciences), and after $30 \mathrm{~min}$ of incubation and washing twice with PBS subsequently centrifuged for $5 \mathrm{~min}$ at $250 \mathrm{~g}$, and cells were resuspended in $500 \mathrm{~mL}$ PBS and filtered through a $100-\mu \mathrm{m}$ strainer. Samples and controls were measured by a BD Flow Cytometer (BD Biosciences), and data analysed using FlowJo 7.6.1 software.

Spheroid culture To prepare $1 \%(\mathrm{w} / \mathrm{v})$ agarose-coated dish, agarose purified powder (BIOWEST, GENE COMPANYLTD, Hongkong, China) was dissolved into phosphate-buffered saline (PBS, Beyotime, Beijing, China). After high-pressure sterilisation, $3 \mathrm{~mL}$ of the agarose solution was added to each $10 \mathrm{~cm}$ culture dish, with gelation at room temperature. For spheroid formation, HDFCs at passage 3 or continuous passage 12 cells in vitro grown as monolayer were dissociated into single cells and seeded into agarose-coated dish at a cell density of approximately 100,000 cells $/ \mathrm{cm}^{2}$, and then incubated at $37^{\circ} \mathrm{C}$ with $5 \% \mathrm{CO}_{2}$ for $4 \mathrm{~d}$. Images of the HDFC spheroids were photographed by phase-contrast microscopy on cultured days 0,2 and 4 , and the diameters of spheroids were measured using ImageJ.

Cell proliferation assay The proliferation of spheroid culture and monolayer culture for later passage (P12) HDFCs was investigated. Spheroids that after $4 \mathrm{~d}$ of culture on $1 \%$ 
agarose-coated dishes were dissociated by trypsin/EDTA, monolayer and spheroid-derived HDFCs were seeded at a density of 2000 cells per well in 96-well plates. Monolayer culture early passage (P3) was a control group. The cell number was measured at $550 \mathrm{~nm}$ by EnSpire Multimode Plate Reader (PerkinElmer, Waltham, MA) every day lasting for 7 $\mathrm{d}$ using the Cell Counting Kit-8 (Beyotime, Beijing, China) according to the manufacturer's instruction.

Real-time PCR Total RNA from monolayer cells or spheroid was extracted using RNAiso Plus reagent (TaKaRa, Shiga, Japan) according to the manufacturer's instructions. Firststrand cDNA was synthesised by PrimeScript ${ }^{\mathrm{TM}}$ RT Master Mix (TaKaRa) according to the manufacturer's protocol. Real-time PCR was performed using a TB Green ${ }^{\circledR}$ Premix Ex Taq ${ }^{\mathrm{TM}}$ II (TaKaRa). CFX Connect Real-time PCR Detection System (BIO-RAD, Hercules, CA) was used to perform and quantify relative amounts of target genes mRNA with glyceraldehyde 3-phosphate dehydrogenase (GAPDH) as an internal control. A primer list used in this study is provided in Table 1.

Western blot The protein expression was determined by western blot. Monolayer and spheroid HDFCs were resuspended in RIPA lysis buffer (Beyotime) and sonicated. After centrifugation, the protein content was determined in the supernatants by a BCA Kit (Beyotime). A total of $30 \mu \mathrm{g}$ of proteins from spheroids or monolayer cells was added and separated by sodium dodecyl sulfate-polyacrylamide gel electrophoresis, and then blotted onto polyvinylidene difluoride membranes. Western blot was performed using anti-Oct4 (1:1000, Cell Signaling Technology, Danvers, MA), antiSox2 (1:1000, Cell Signaling Technology), anti-Nanog (1:1000, Cell Signaling Technology), anti-ALP (1:200, Santa Cruz Biotechnology, Santa Cruz, CA), anti-OPN (1:200, Santa Cruz Biotechnology), anti-ON (1:200, Santa Cruz Biotechnology), anti-BSP (1:200, Santa Cruz
Biotechnology), anti-DSPP (1:200, Santa Cruz Biotechnology), anti-DMP-1 (1:200, Abcam, Cambridge, MA) and anti-GAPDH (1:2000, Santa Cruz Biotechnology). The membranes were incubated with the primary antibodies overnight at $4^{\circ} \mathrm{C}$. After extensive washing, the membranes were further incubated with horseradish peroxidaseconjugated secondary antibodies (1:4000, Abcam) for $1 \mathrm{~h}$. The blots were visualised using an enhanced chemiluminescence BeyoECL star detection kit (Beyotime) and ChemiDoc ${ }^{\mathrm{TM}}$ Imaging System quantified band intensities.

Immunofluorescence staining After $4 \mathrm{~d}$, spheroid formation or monolayer on slides was washed with PBS and fixed in 4\% paraformaldehyde at room temperature for $10 \mathrm{~min}$, after three times washed with PBST, and permeabilised with $0.1 \%$ Triton $\mathrm{X}-100$ solution for $15 \mathrm{~min}$ at room temperature. Then, washed with PBST, primary antibodies were then incubated with samples overnight at $4^{\circ} \mathrm{C}$ : anti-Oct 4 (1:200, Cell Signaling Technology), anti-Sox 2(1:200, Cell Signaling Technology), anti-Nanog (1:200, Cell Signaling Technology), anti-CK14 (1:200, ZSGB-BIO, Beijing, China), anti-Vimentin (1:200, ZSGB-BIO), anti-DSPP (1:100, Santa Cruz Biotechnology) and anti-DMP-1 (1:100, Abcam). After incubation with primary antibodies, samples were washed with PBST and then incubated with goat anti-rabbit IgG/RBITC or goat antimouse IgG/RBITC (1:100, Solarbio Life Science, Beijing, China) for $1 \mathrm{~h}$ at room temperature. For nuclear DNA dye, the samples were mounted with DAPI. Images were captured using the Leica TCS SP8 laser scanning confocal microscopy system.

Alizarin red staining and quantification of calcification After $21 \mathrm{~d}$ of induction culture, cells from each group were rinsed three times with PBS, fixed in $4 \%$ paraformaldehyde solution at room temperature for $15 \mathrm{~min}$ and washed three times with distilled water. Then, the cells were incubated with $0.2 \%$ Alizarin red solution

Table 1 Sequences of primers used for qRT-PCR in this study

\begin{tabular}{lll}
\hline Gene & Forward & Reverse \\
\hline Sox2 & 5'-TCAGGAGTTGTCAAGGCAGAGAAG-3' & 5'-GCCGCCGCCGATGATTGTTATTAT-3' \\
Oct4 & 5'-GATGGCGTACTGTGGGCCC-3' & 5'-CAAAACCCGGAGGAGTCCCA-3' \\
Nanog & 5'-AACGGCCTGACTCAGAAGGGCT-3' & 5'-AAGGTTTCCAGACGCGTTCATCA-3' \\
ALP & 5'-TAAGGACATCGCCTACCAGCTC-3' & 5'-TCTTCCAGGTGTCAACGAGGT-3'-3' \\
DSPP & 5'-CTGTTGGGAAGAGCCAAGATAAG-3' & 5'-CCAAGATCATTCCATGTTGTCCT-3' \\
DMP-1 & 5'-GTGAGTGAGTCCAGGGGAGATAA-3' & 5'-TTTTGAGTGGGAGAGTGTGTGC-3' \\
ON & 5'-CCCATTGGCGAGTTTGAGAAG-3' & 5'-CAAGGCCCGATGTAGTCCA-3' \\
OPN & 5'-CAGTTGTCCCCACAGTAGACAC-3' & 5'-GTGATGTCCTCGTCTGTAGCATC-3' \\
BSP & 5'-GATTTCCAGTTCAGGGCAGTAG-3' & 5'-CCCAGTGTTGTAGCAGAAAGTG-3' \\
GAPDH & 5'-TGCACCACCAACTGCTTAGC-3' & 5'-GGCATGGACTGTGGTCATGAG-3' \\
\hline
\end{tabular}


(Solarbio, Beijing, China) for $30 \mathrm{~min}$ at room temperature, removing dye solution, and washed with deionised water three times. Images were taken by a microscope, to quantify the degree of calcification by using ImageJ software.

Statistical analysis All measurements are presented as means \pm SD. Statistical significance was evaluated using an independent-samples Student $t$ test; two-tailed Student's $t$ test was used to detect the differences between the groups. All statistical analyses were performed using Graph Pad Prism 5. Statistically significant values were defined as $* p<0.05$ and $* * p<0.01$.

\section{Results}

Isolation and identification of HDFCs The primary HDFCs were isolated and cultured; the adherent cells exhibited MSC typical spindle-shaped and fibroblast-like morphology (Fig. $1 A-C$ ). The immunofluorescence staining results exhibited positively expressed mesenchymal cell marker vimentin but no expression of the epithelial cell marker CK14 (Fig. 1D). The surface marker expression characteristics were also analysed using flow cytometry, as the results demonstrated that HDFCs showed positive expression of the mesenchymal surface markers CD90 and CD105 but lacked expression of CD34 and CD45, suggesting HDFCs lack haematopoietic lineages (Fig. 1E). These results indicate that $\mathrm{HDFC}$ retain mesenchymal phenotypes and mesenchymal stem cell characteristics.

Spheroid culture of HDFCs can partially restore the activity of later passage cell proliferation HDFC primary clusters were seeded to $1 \%$ agarose-coated dishes and cultured to form HDFC spheroid (Fig. 2A). HDFCs plated on the $1 \%$ agarose-coated dishes did not attach to the dish surface but formed spheroids; field microscopy observes showed that small spheroids with different sizes close to the shape of a sphere formed at day 2, and the diameter of HDFC spheroid expanded to 20 to $150 \mu \mathrm{m}$ after $4 \mathrm{~d}$ (Fig. $2 B$ ). In addition, we found that those HDFCs expanding continuously for 12 generations showed an ageing phenotype and were challenging to expand, but the 3 passages of HDFCs maintained high cell
A

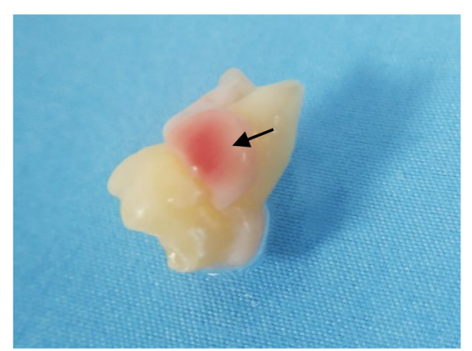

B

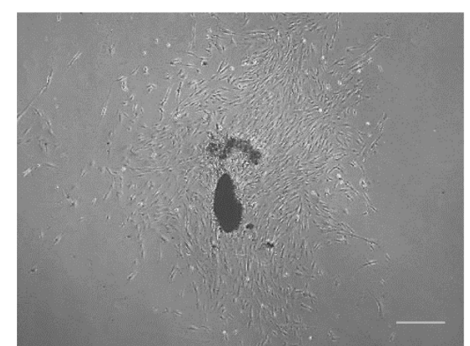

C

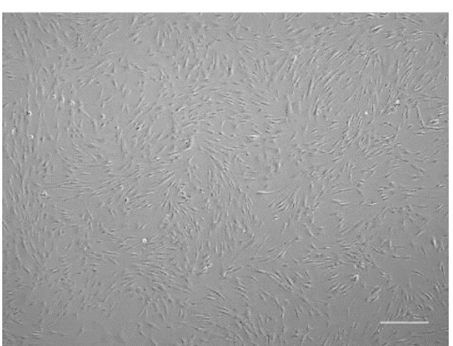

D
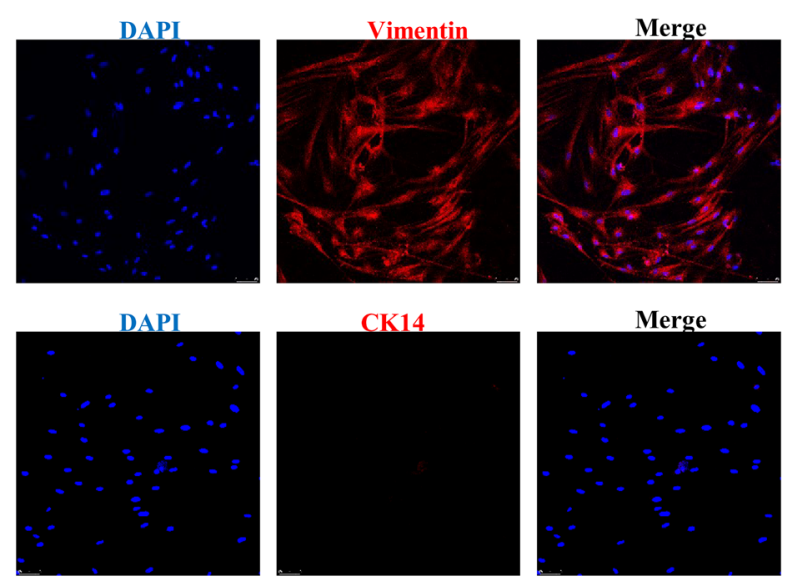

Fig. 1. Isolation and characterisation of human dental follicle cells. (A) Isolated third molars and dental follicles (arrow). $(\boldsymbol{B}, \boldsymbol{C})$ Representative morphology images of HDFC passage 1 and passage 3 . (D)

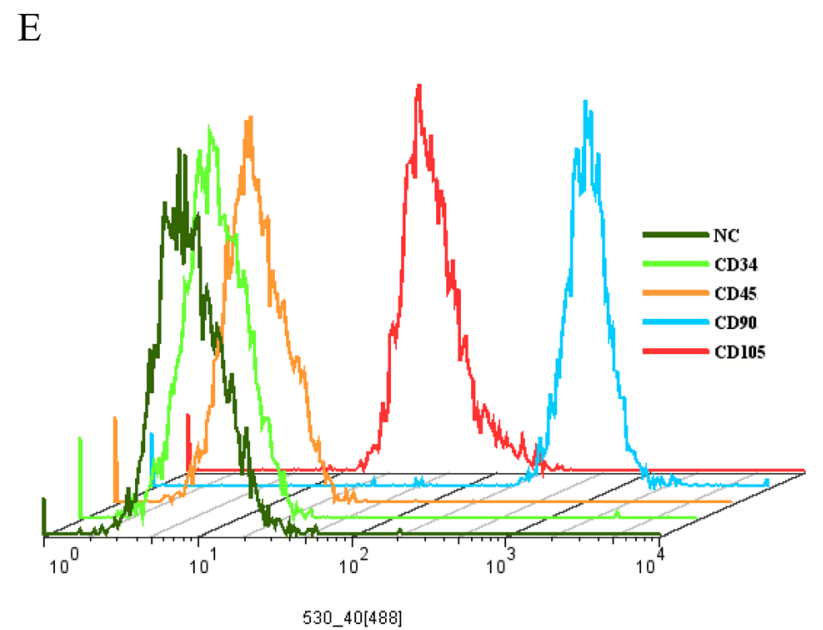

Immunocytofluorescence staining for vimentin and CK14 at passage 3 . (E) Representative flow cytometry analysis of HDFCs for surface markers CD90, CD105, CD34 and CD45. 
A

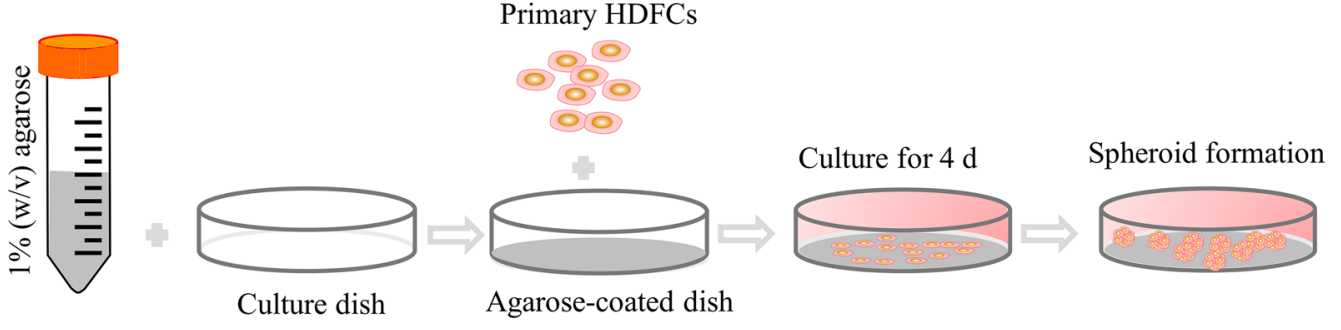

B

Day 0

Day 2

Day 4
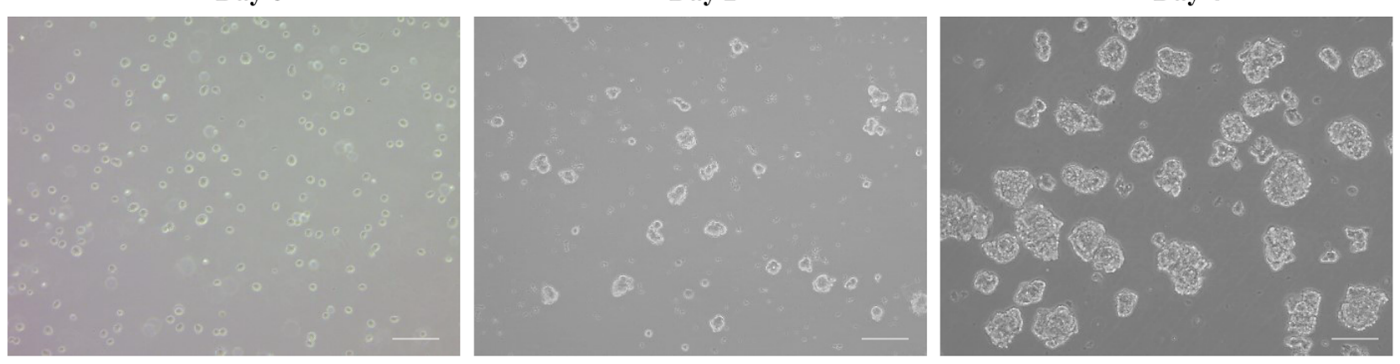

$\mathrm{C}$

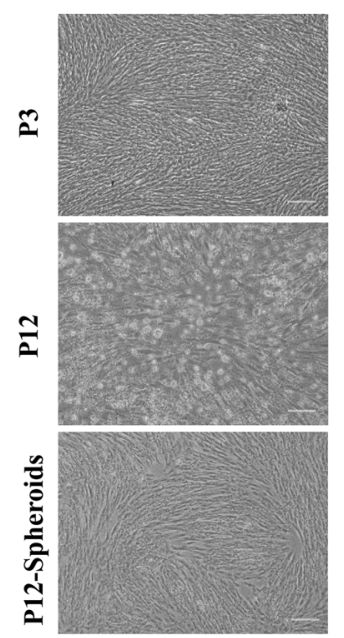

D

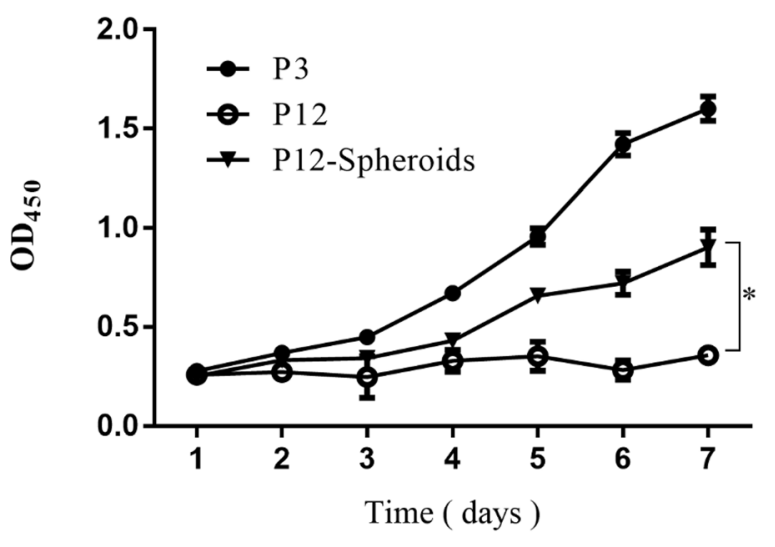

Fig. 2. Characteristics of HDFC spheroid formation and HDFC proliferative ability of early passage or derived from late passage spheroids. (A) The technology used for the generation of HDFC spheroids. (B) The growing process captured for morphology of spheroid culture formation was observed at day 0 , day 2 and day 4 . The scale bar is $100 \mathrm{~mm}$. (C) The morphology of HDFCs grown in

monolayer culture at passage $3(\mathrm{P} 3)$ and passage 12 (P12) or the HDFCs derived from spheroid formation from passage 12 cultures (P12-spheroids). Scale bar: $100 \mu \mathrm{m}$. (D) Growth curves of HDFCs derived from monolayer and spheroid cultures at the initial seeding density of $5 \times 10^{4}$ cells $/ \mathrm{mL}$.

viability. However, those cells derived from late passage HDFC spheroids (P12-spheroids) can restore proliferation (Fig. 2C). Moreover, the CCK-8 cell proliferation assay observed that spheroid culture conditions significantly restored the viability of the later passages of HDFCs and promoted their proliferation but exhibited decreased viability compared to the early passage cells (Fig. 2D). Our results suggest that the HDFCs from spheroid suspension culture can partially restore the proliferation ability of later passages of HDFCs.

Enhancement of stemness in HDFCs under spheroid culture Sox2, Oct4 and Nanog are important transcription factors for maintaining the pluripotency and stemness of stem cells. The qRT-PCR analysis showed that the mRNA levels of pluripotency genes Sox2, Nanog and Oct4 in HDFC spheroids cultured were significantly higher than those in the monolayer-cultured group (Fig. 3A). The protein level analysed by western blot showed that Sox2, Oct4 and Nanog protein level was higher in spheroids than those cells in the monolayer culture (Fig. $3 B$ ). Immunofluorescence staining also showed that the HDFC spheroids were positive for Sox2, Oct4 and Nanog, whereas monolayer culture showed lower or no expression for these markers (Fig. 
A

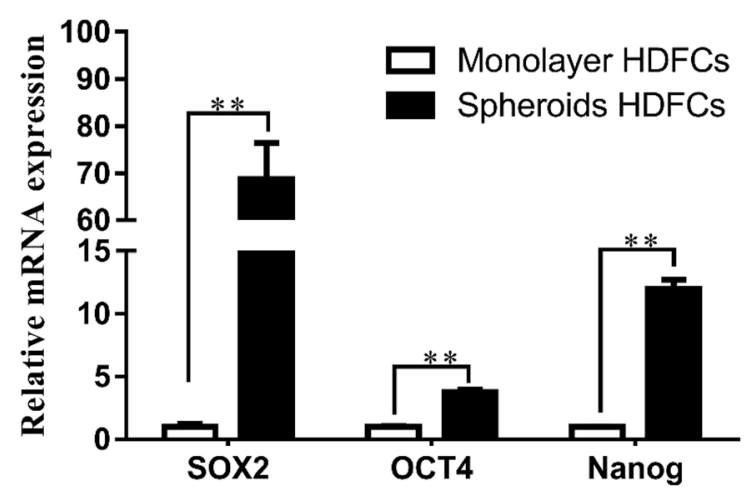

B

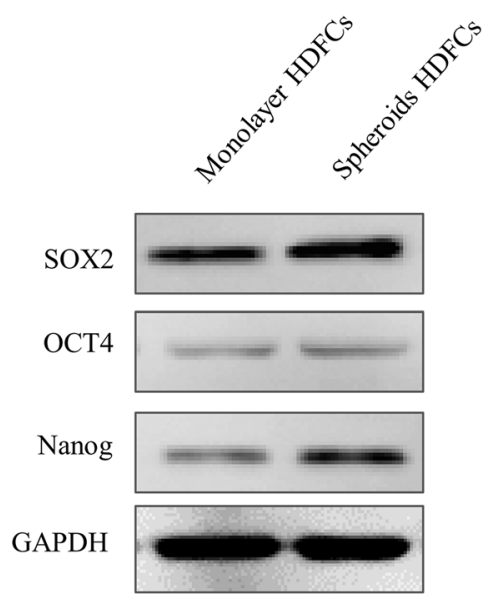

Fig. 3. Enhanced expression of stemness markers in HDFC spheroids. (A) The mRNA expression analysis via qRT-PCR for pluripotency marker genes in HDFC spheroids after $4 \mathrm{~d}$ of culture on $1 \%$ agarose-coated dishes $(* * p<0.01$, unpaired Student's $t$ test, $\mathrm{n}=3$ ). (B) Western blot

3C). These data indicate that HDFC spheroid culture can promote the expression of pluripotent markers and enhance stemness properties compared with monolayer culture.

Odonto/osteogenic differentiation of cells from DFSCs spheroid In order to compare odonto/osteogenic differentiation potential, we seeded DFSC spheroids or monolayer-cultured DFSCs on culture plates in odonto/osteogenic induction medium. DFSC spheroid adhered and grew into monolayers. After $14 \mathrm{~d}$ of culture in induction medium, compared with monolayer HDFCs, the qRT-PCR analysis showed that the odontogenic differentiation-related genes significantly increased and osteogenic marker genes decreased in HDFC spheroids (Fig. 4A). Western blot analysis also revealed a high expression of odontogenic differentiation markers DMP-1 and DSPP, whereas the osteogenic markers ALP, BSP, ON and OPN in HDFC spheroids induced by odonto/osteogenic
C
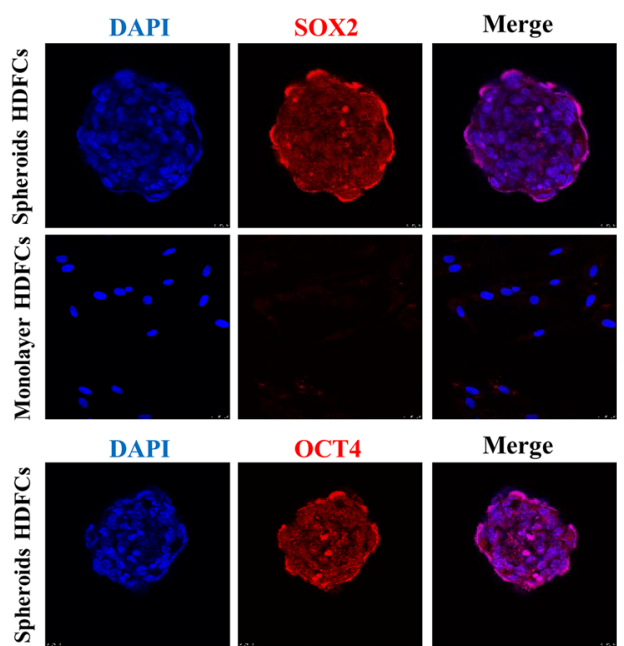

Merge
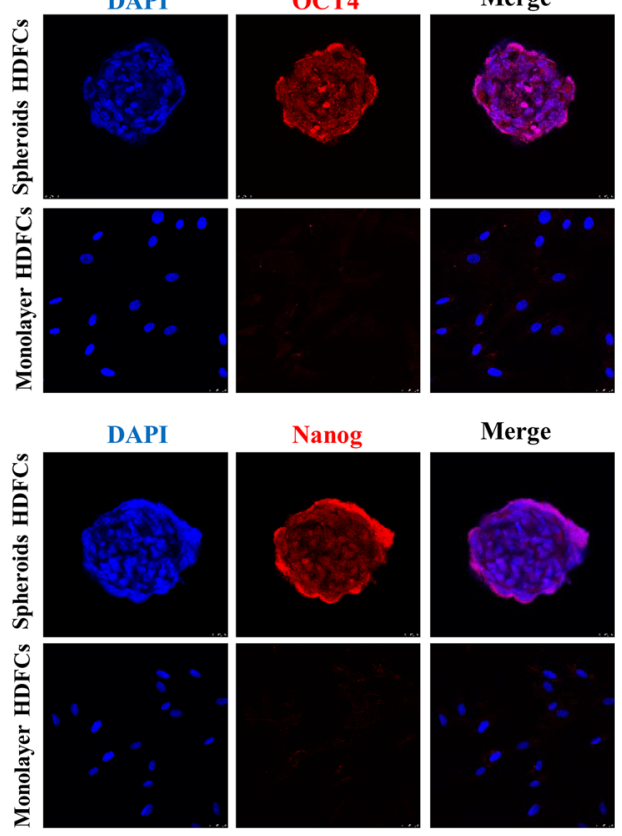

assays of the protein level for pluripotency factors in HDFC spheroids and monolayer. $(\boldsymbol{C})$ Representative images of phenotype by immunofluorescence staining in HDFC spheroids.

induction medium were weakly expressed (Fig. $4 B$ ). In addition, immunofluorescence staining demonstrated that the odontogenic differentiation-related genes DMP-1 and DSPP were positive and more expressed in HDFC spheroids of differentiation with odonto/osteogenic induction medium (Fig. $4 C)$. After $21 \mathrm{~d}$ of culture in odonto/osteogenic induction medium, mineralised nodules were detected by Alizarin red $\mathrm{S}$ staining. Alizarin red mineralised nodules were markedly increased in the HDFC spheroids compared with those from monolayer culture (Fig. 4D). These data demonstrate that HDFC spheroids significantly promoted odontogenic differentiation and prevented osteogenic induction in vitro.

\section{Discussion}

Traditionally, bone marrow (BM) has been the prevailing source of MSC in humans. Cells from the BM can be 
A

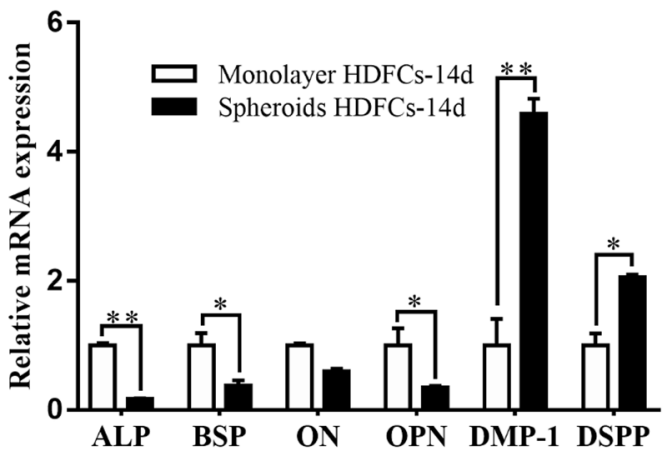

B

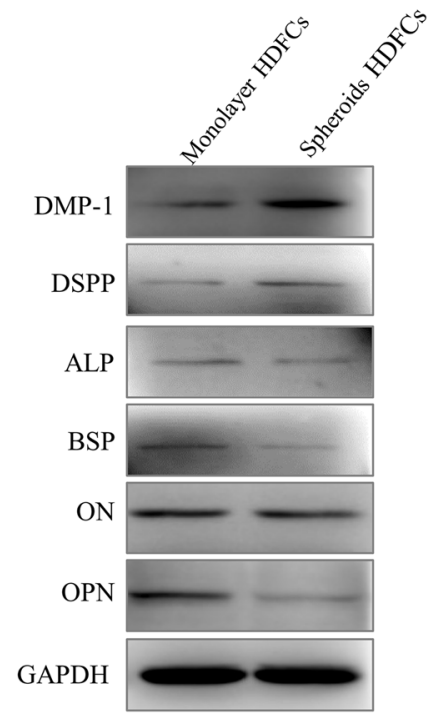

C
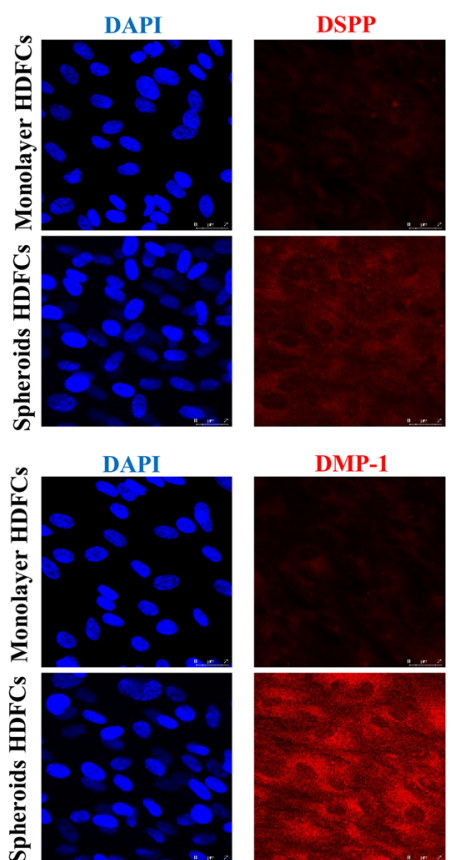

DMP-1
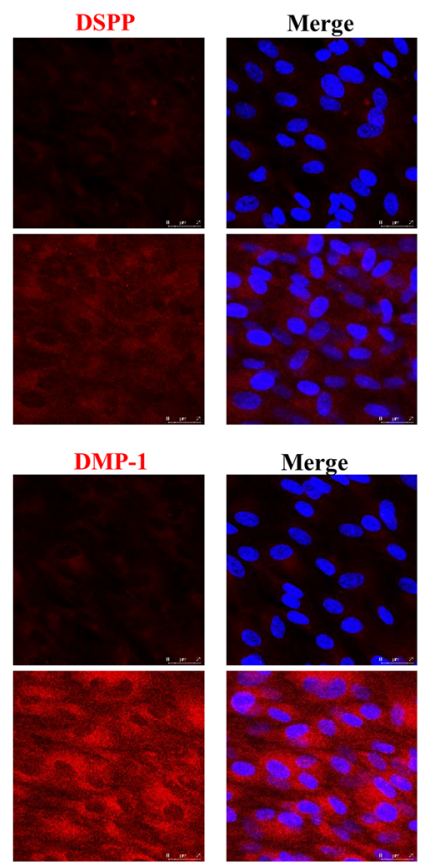

$\mathrm{D}$ Monolayer HDFCs Spheroids HDFCs
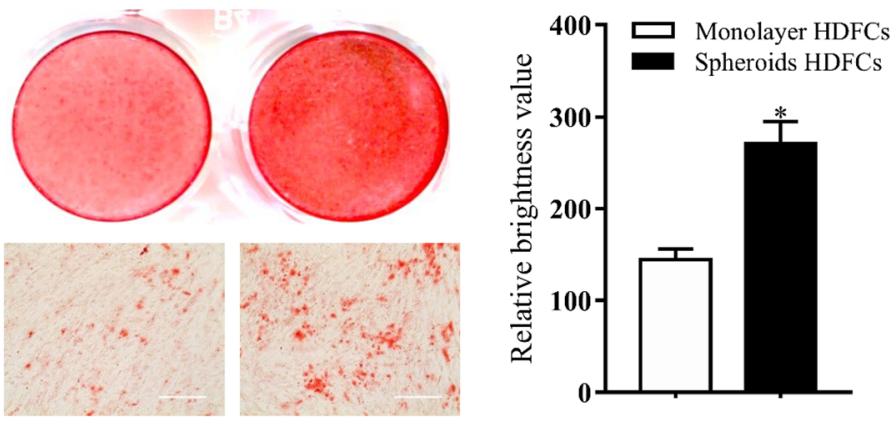

Fig. 4. Enhancement of odontogenic differentiation in HDFCs from spheroid culture in vitro. (A) Quantitative RT-PCR analysis of odonto/ osteogenic differentiation marker gene expression levels after induction for 14 d. (B) Expression of odonto/osteogenic differentiation marker gene in spheroid and monolayer HDFCs analysed by western blot after induction for $14 \mathrm{~d}$. (C) Immunofluorescence staining of odontogenic

induced to multilineage including osteogenic, chondrogenic and adipogenic differentiation potential (Pittenger, et al. 1999). However, commonly and traditionally isolated MSCs from bone marrow, adipose tissue and umbilical cord blood can present many practical limitations, including the difficulty and the invasiveness of the isolation procedures (Berebichez-Fridman and MonteroOlvera, 2018). Dental MSCs, which share properties with MSCs, can be isolated with ease from distinct dental/oral tissues, making them an attractive source of autologous stem cells for broad application in regenerative medicine (Volponi and Sharpe, 2013). These dental MSCs can be obtained from different dental tissues, such as the

differentiation maker genes DSPP and DMP-1. (D) Microscopic images of monolayer and spheroid-derived HDFCs cultured in osteogenic induction media for $21 \mathrm{~d}$. Calcified nodule formation was stained with Alizarin red, and quantification analyses of Alizarin red were performed. Scale bars $=100 \mu \mathrm{m} . * p<0.05, * * p<0.01$.

periapical cyst, exfoliated deciduous teeth (Miura, et al. 2003), dental follicle (Morsczeck, et al. 2005), periodontal ligament (Seo, et al. 2004), root apical papilla (Sonoyama, et al. 2008) and dental pulp (Gronthos, et al. 2000). Dental MSCs have been found to have easy accessibility, genomic stability, immunomodulatory features and multilineage differentiation capacities; all these characteristics of dental MSCs make them as a precious stem cell source for regenerative medicine and stem cell-based therapy (Andrukhov, et al. 2019, Gan, et al. 2020). Among the dental tissues, the dental follicle surrounds the tooth germ and can be easily separated from extracted wisdom teeth. The stem cells from this tissue (DFCs) comprise a cell mixture of 
heterogeneous populations of stem cells and progenitor cells for cementoblasts, periodontal ligament cells and osteoblasts (Morsczeck, et al. 2005). It is believed that HDFCs with multiple differentiation potential have great potential for both dentin formation and regeneration. However, maintaining the stemness properties and enhancing the therapeutic potential of HDFCs during traditional adherent culture are still challenging.

In this study, the HDFCs were separated from impacted third molars, and the fibroblast-like and spindle-shaped morphology was observed and similar to previous reports. Immunofluorescence staining showed the positive expression of the mesenchymal cell marker vimentin, flow cytometry analysis showed that HDFCs displayed expression of the mesenchymal stem cell markers CD146 and CD90, but did not express the haematopoietic lineage markers CD34 and CD45. Our observations showed that HDFCs have high proliferative activity, but cell proliferation decreased significantly. In addition, cells were challenging to be subcultured after 12 continuous passages in vitro. The later passage HDFCs exhibited secrete senescence-associated secretory phenotype (SASP), which is a characteristic of cellular senescence (Malaquin, et al. 2016). During in vitro expansion on two-dimensional (2D) plastic culture plates, MSC proliferation is limited by the cellular senescence and lose their stemness properties and differentiation capacity. Cells usually require biological signals from the cellular niches, encouraging proliferation and enhancing cellular viability (Cui, et al. 2017, Ryu, et al. 2019).

To overcome the limitations associated with 2D culture, 3D cell culture models were developed to create tissue-like structures and recreate a more physiologically relevant environment (Abbott 2003, Knight and Przyborski, 2015). So far, numerous 3D in vitro models have been developed, such as cellular organoids (Kratochvil, et al. 2019), spheroids (Lee, et al. 2018), cell-laden biomimetic constructs (Koo, et al. 2018) and mini-organs (Noor, et al. 2019). Spheroids are formed via one or more cell types proliferating, differentiating and self-organising within close proximity to one another. Compared with traditional monolayer culture system, 3D spheroids maintain their intrinsic phenotypic properties by cell-extracellular matrix interactions, and this technique is a promising three-dimensional cell culture method showing applicability in various fields such as cancer research, stem cell biology and regenerative medicine (Cesarz and Tamama, 2016. Previous reports have established forming hepatocyte spheroids by using agarose-coated dishes (Hasebe, et al. 2005). In this study, agarose was used as biomaterial to construct a 3D structure of HDFC spheroids as it is natural, low cost and non-toxic. We applied the agarose culture method to HDFC spheroid formation for the first time. The HDFCs plated on $1 \%$ agarose-coated dish rapidly self-assembled to form a cubical morphology, and then, formed spheroids have different sizes; the diameter of the sphere is between 20 and 150 microns (Fig. 2). A recent study used agarose for a microwellbased approach to prepare size-controlled and highly viable glioma spheroids, and the final size of these homogeneous spheroids depended on cell seeding density and microwell size (Mirab, et al. 2019). Together, these results demonstrate that agarose is a practical material for $3 \mathrm{D}$ cell culture for HDFC spheroid formation and has therapeutic potential for tissue engineering.

HDFCs are regarded as the ideal cell source for tissue regeneration and regenerative medicine due to it being easily isolated from discarded teeth. A previous study showed that DFCs could easily be cultured for more than 6 passage (Morsczeck, et al. 2005). However, after long-term culture in vitro, cell proliferation decreased significantly and finally stopped (Morsczeck, et al. 2019). Therefore, cell senescence and loss of differentiation potentials compromised HDFC clinical application. In this study, our results show that HDFCs within spheroids can be further expanded in monolayer culture.

Moreover, another interesting feature of HDFC spheroids is that spheroidal formation can restore cell senescence properties of late passage (passage 12) of HDFCs in vitro. Spheroid-derived late passage HDFCs exhibited significantly increased proliferation ability (Fig. 2), consistently with previous studies. A study showed that senescence was significantly decreased in spheroid culture of adipose-derived stem cells in vitro, compared with monolayer cells (Cheng, et al. 2013). A recent study demonstrated that late passage ASC cellular senescence can be reversed through co-culture with ECFCs in vitro (Hu, et al. 2020). In addition, proliferation comparison of late passage HDFC spheroid-derived cells and early passage HDFCs demonstrated that HDFCs derived from spheroid formation by passage 12 displayed lower proliferation than passage 3 . This suggests that cell senescence is caused by long-term culture in vitro and that it is an irreversible process.

Several recent reports have demonstrated an increased stemness property by spheroid formation culture. The 3D spheroid MSC culture dramatically upregulates stemness markers compared with the 2D monolayer MSC culture and contributes to a shorter regenerative healing process, including new bone formation (Imamura, et al. 2020). Consistent with previous studies, this study also shows the spheroidal formation increased gene expression of pluripotency marker genes such as Nanog, Sox 2 and Oct4. To explore the role of spheroid culture methods in the odontogenic differentiation of HDFCs, we examined the gene expression of ALP, ON, OPN and BSP as osteogenic differentiation markers, and DSPP and DMP-1 as odontogenic differentiation markers. DSPP and DMP1 play an important role during early odontoblastic differentiation and late dentin mineralization, and high levels of expression of ALP, OPN and ON regard to early/late osteoblastic differentiation (Ching, et al. 2017). The formation of 
calcified nodules was also examined. It is now obviously accepted that ALP, DSPP and DMP-1 play important roles in hard tissue mineralization (Suzuki, et al. 2012, Schutte, et al. 2013). Previous studies have indicated that DSPP is a downstream effector molecule of DMP1 and is essential for dentin formation (George, et al. 1993, Gibson, et al. 2013). DFCs, as a prospective seeding cell, exhibited strong osteogenesis and adipogenesis capacities (Yang, et al. 2019). This study found that the expression levels of odontogenic-related genes DSPP and DMP1 were significantly increased in the spheroid culture HDFC group. Compared with monolayer-cultured HDFCs, the expression of osteogenesis-related genes ALP, ON, OPN and BSP was significantly downregulated in HDFC spheroids. Contrary to previous studies, spheroid culture is reported to enhance osteogenic differentiation potential such as periodontal ligament mesenchymal stem cells (Moritani, et al. 2018) and mesenchymal stem cells (Ho, et al. 2018). These results suggest that spheroid cell culture positively affects the odontogenic differentiation of HDFCs in vitro. Further research is needed to explore the mechanisms of how the spheroid culture reverses late passage HDFC senescence and promotes odontogenic differentiation. This study highlights the importance of maintaining stemness and enhanced odontogenic differentiation through spheroid culture by agarose in vitro.

We also found the more calcification in spheroid-cultured HDFCs during mineralization-inducing medium in vitro, while lower ALP expression compared to monolayercultured HDFC cells. ALP plays a role in hydrolyses pyrophosphate and supplies adequate inorganic phosphate to enhance mineralization. The in vitro study suggested that DSPP induces organised mineralization of collagen fibrils and DMP1 induces the deposition of organised mineral bundles (Deshpande, et al. 2011). Previous reports also disclosed that NIH3T3 cells over-expressing PP (dentin phosphoprotein or phosphophoryn) showed extensive mineral deposition after culture in mineralization-inducing medium (Sfeir, et al. 2011). These results indicate that the function of DSPP and DMP-1 was an inducer of mineralization. One possible explanation is that the biomineralization in the odontoblast differentiation more depended on DSPP and DMP-1 expression rather than ALP in spheroid-cultured HDFCs. However, further analysis is required to determine the molecular mechanisms that DSPP and DMP-1 play in biomineralization of spheroid-cultured HDFCs. Further study should also analyse whether this biomineralization process requires the involvement of ALP in odontoblast cell differentiation.

\section{Conclusion}

In summary, we report a simple method to produce HDFC spheroid. HDFC spheroid-derived cells also exhibit a partial restored proliferative activity of late passage senescent cells in vitro. The stemness properties were enhanced by the spheroid formation of HDFCs in agarose. Moreover, HDFC spheroids significantly promoted odontogenic differentiation in vitro. Our results demonstrated that HDFC spheroidderived cells might be a valuable resource for application in tooth regeneration.

Funding Funding for this project was supported by the National Natural Science Foundation of China (81600819) and Department of Science and Technology of Sichuan Province (2018FZ0113).

\section{Declarations}

Conflict of interest The authors declare no competing interests.

\section{References}

Abbott A (2003) Cell culture: biology's new dimension. Nature 424:870872

Andrukhov O, Behm C, Blufstein A, Rausch-Fan X (2019) Immunomodulatory properties of dental tissue-derived mesenchymal stem cells: implication in disease and tissue regeneration. World J Stem Cells 11:604-617

Baraniak PR, McDevitt TC (2012) Scaffold-free culture of mesenchymal stem cell spheroids in suspension preserves multilineage potential. Cell Tissue Res 347:701-711

Bartosh TJ, Ylostalo JH, Mohammadipoor A, Bazhanov N, Coble K, Claypool K, Lee RH, Choi H, Prockop DJ (2010) Aggregation of human mesenchymal stromal cells (MSCs) into 3D spheroids enhances their antiinflammatory properties. P Natl Acad Sci USA 107: 13724-13729

Berebichez-Fridman R, Montero-Olvera PR (2018) Sources and clinical applications of mesenchymal stem cells: state-of-the-art review. Sultan Qaboos Univ Med J 18:e264-e277

Cesarz Z, Tamama K (2016) Spheroid culture of mesenchymal stem cells. Stem Cells Int 2016:9176357

Charriere K, Risold PY, Fellmann D (2010) In vitro interactions between bone marrow stromal cells and hippocampal slice cultures. Cr Biol 333:582-590

Cheng NC, Chen SY, Li JR, Young TH (2013) Short-term spheroid formation enhances the regenerative capacity of adipose-derived stem cells by promoting stemness, angiogenesis, and chemotaxis. Stem Cells Transl Med 2:584-594

Cheng NC, Wang S, Young TH (2012) The influence of spheroid formation of human adipose-derived stem cells on chitosan films on stemness and differentiation capabilities. Biomaterials 33:17481758

Ching HS, Luddin N, Rahman IA, Ponnuraj KT (2017) Expression of Odontogenic and Osteogenic Markers in DPSCs and SHED: A Review. Current stem cell research \& therapy 12:71-79

Cui X, Hartanto Y, Zhang H (2017) Advances in multicellular spheroids formation. J R Soc Interface 14:20160877

Deshpande AS, Fang PA, Zhang X, Jayaraman T, Sfeir C, Beniash E (2011) Primary structure and phosphorylation of dentin matrix protein 1 (DMP1) and dentin phosphophoryn (DPP) uniquely determine their role in biomineralization. Biomacromolecules 12:29332945

Discher DE, Mooney DJ, Zandstra PW (2009) Growth factors, matrices, and forces combine and control stem cells. Science 324:1673-1677 
Fu T, Liang P, Song J, Wang J, Zhou P, Tang Y, Li J, Huang E (2019) Matrigel scaffolding enhances BMP9-induced bone formation in dental follicle stem/precursor cells. Int J Med Sci 16:567-575

Fukuda J, Khademhosseini A, Yeo Y, Yang XY, Yeh J, Eng G, Blumling J, Wang CF, Kohane DS, Langer R (2006) Micromolding of photocrosslinkable chitosan hydrogel for spheroid microarray and co-cultures. Biomaterials 27:5259-5267

Gan L, Liu Y, Cui D, Pan Y, Zheng L, Wan M (2020) Dental tissuederived human mesenchymal stem cells and their potential in therapeutic application. Stem Cells Int 2020:8864572

George A, Sabsay B, Simonian PA, Veis A (1993) Characterization of a novel dentin matrix acidic phosphoprotein. Implications for induction of biomineralization J Biol Chem 268:12624-12630

Gibson MP, Zhu QL, Wang SZ, Liu QL, Liu Y, Wang XF, Yuan BZ, Ruest LB, Feng JQ, D'Souza RN, Qin CL, Lu YB (2013) The rescue of dentin matrix protein 1 (DMP1)-deficient tooth defects by the transgenic expression of dentin sialophosphoprotein (DSPP) indicates that DSPP is a downstream effector molecule of DMP1 in dentinogenesis. J Biol Chem 288:7204-7214

Gronthos S, Mankani M, Brahim J, Robey PG, Shi S (2000) Postnatal human dental pulp stem cells (DPSCs) in vitro and in vivo. Proc Natl Acad Sci U S A 97:13625-13630

Hasebe Y, Okumura N, Koh T, Kazama H, Watanabe G, Seki T, Ariga T (2005) Formation of rat hepatocyte spheroids on agarose. Hepatol Res 32:89-95

Ho SS, Hung BP, Heyrani N, Lee MA, Leach JK (2018) Hypoxic preconditioning of mesenchymal stem cells with subsequent spheroid formation accelerates repair of segmental bone defects. Stem Cells 36:1393-1403

Hu W, Zhu S, Fanai ML, Wang J, Cai J, Feng J (2020) 3D co-culture model of endothelial colony-forming cells (ECFCs) reverses late passage adipose-derived stem cell senescence for wound healing. Stem Cell Res Ther 11:355

Huang GS, Dai LG, Yen BL, Hsu SH (2011) Spheroid formation of mesenchymal stem cells on chitosan and chitosan-hyaluronan membranes. Biomaterials 32:6929-6945

Imamura A, Kajiya H, Fujisaki S, Maeshiba M, Yanagi T, Kojima H, Ohno J (2020) Three-dimensional spheroids of mesenchymal stem/ stromal cells promote osteogenesis by activating stemness and Wnt/ beta-catenin. Biochem Biophys Res Commun 523:458-464

Knight E, Przyborski S (2015) Advances in 3D cell culture technologies enabling tissue-like structures to be created in vitro. J Anat 227:746756

Koo Y, Choi EJ, Lee J, Kim HJ, Kim G, Do SH (2018) 3D printed cellladen collagen and hybrid scaffolds for in vivo articular cartilage tissue regeneration. J Ind Eng Chem 66:343-355

Kratochvil MJ, Seymour AJ, Li TL, Pasca SP, Kuo CJ, Heilshorn SC (2019) Engineered materials for organoid systems. Nat Rev Mater 4: 606-622

Kuwashima Y, Yamada T, Saio M, Takami T (1993) Formation and growth of multicellular spheroids in media containing low concentrations of agarose. Cancer Lett 71:31-33

Lee YB, Kim EM, Byun H, Chang HK, Jeong K, Aman ZM, Choi YS, Park J, Shin H (2018) Engineering spheroids potentiating cell-cell and cell-ECM interactions by self-assembly of stem cell microlayer. Biomaterials 165:105-120

Levy O, Kuai R, Siren EMJ, Bhere D, Milton Y, Nissar N, De Biasio M, Heinelt M, Reeve B, Abdi R, Alturki M, Fallatah M, Almalik A, Alhasan AH, Shah K, Karp JM (2020) Shattering barriers toward clinically meaningful MSC therapies. Sci Adv 6:eaba6884
Malaquin N, Martinez A, Rodier F (2016) Keeping the senescence secretome under control: molecular reins on the senescenceassociated secretory phenotype. Exp Gerontol 82:39-49

McKee C, Chaudhry GR (2017a) Advances and challenges in stem cell culture. Colloids Surf B: Biointerfaces 159:62-77

McKee C, Chaudhry GR (2017b) Advances and challenges in stem cell culture. Colloid Surface B 159:62-77

Miao Y, Sun YB, Liu BC, Jiang JD, Hu ZQ (2014) Controllable production of transplantable adult human high-passage dermal papilla spheroids using 3D Matrigel culture. Tissue Eng Pt A 20:23292338

Mirab F, Kang YJ, Majd S (2019) Preparation and characterization of size-controlled glioma spheroids using agarose hydrogel microwells. Plos One 14

Miura M, Gronthos S, Zhao M, Lu B, Fisher LW, Robey PG, Shi S (2003) SHED: stem cells from human exfoliated deciduous teeth. Proc Natl Acad Sci U S A 100:5807-5812

Moritani Y, Usui M, Sano K, Nakazawa K, Hanatani T, Nakatomi M, Iwata T, Sato T, Ariyoshi W, Nishihara T, Nakashima K (2018) Spheroid culture enhances osteogenic potential of periodontal ligament mesenchymal stem cells. J Periodontal Res 53:870-882

Morrison SJ, Spradling AC (2008) Stem cells and niches: mechanisms that promote stem cell maintenance throughout life. Cell 132:598 611

Morsczeck C, Gotz W, Schierholz J, Zeilhofer F, Kuhn U, Mohl C, Sippel C, Hoffmann KH (2005) Isolation of precursor cells (PCs) from human dental follicle of wisdom teeth. Matrix Biol 24:155-165

Morsczeck C, Reck A, Reichert TE (2019) Short telomeres correlate with a strong induction of cellular senescence in human dental follicle cells. BMC Mol Cell Biol 20:5

Mushahary D, Spittler A, Kasper C, Weber V, Charwat V (2018) Isolation, cultivation, and characterization of human mesenchymal stem cells. Cytometry A 93:19-31

Noor N, Shapira A, Edri R, Gal I, Wertheim L, Dvir T (2019) 3D Printing of personalized thick and perfusable cardiac patches and hearts. Advanced science 6

Pittenger MF, Mackay AM, Beck SC, Jaiswal RK, Douglas R, Mosca JD, Moorman MA, Simonetti DW, Craig S, Marshak DR (1999) Multilineage potential of adult human mesenchymal stem cells. Science 284:143-147

Ryu NE, Lee SH, Park H (2019) Spheroid culture system methods and applications for mesenchymal stem cells. Cells 8

Schutte R, Huisman HW, Malan L, van Rooyen JM, Smith W, Glyn MC, Mels CM, Fourie CM, Malan NT, Schutte AE (2013) Alkaline phosphatase and arterial structure and function in hypertensive African men: the SABPA study. Int J Cardiol 167:1995-2001

Seo BM, Miura M, Gronthos S, Bartold PM, Batouli S, Brahim J, Young M, Robey PG, Wang CY, Shi S (2004) Investigation of multipotent postnatal stem cells from human periodontal ligament. Lancet 364 : $149-155$

Sfeir C, Lee D, Li J, Zhang X, Boskey AL, Kumta PN (2011) Expression of phosphophoryn is sufficient for the induction of matrix mineralization by mammalian cells. J Biol Chem 286:20228-20238

Sonoyama W, Liu Y, Yamaza T, Tuan RS, Wang S, Shi S, Huang GT (2008) Characterization of the apical papilla and its residing stem cells from human immature permanent teeth: a pilot study. J Endod 34:166-171

Suzuki S, Haruyama N, Nishimura F, Kulkarni AB (2012) Dentin sialophosphoprotein and dentin matrix protein-1: two highly phosphorylated proteins in mineralized tissues. Arch Oral Biol 57:11651175 
Tang YD, Liu JM, Chen Y (2016) Agarose multi-wells for tumour spheroid formation and anti-cancer drug test. Microelectron Eng 158:4145

Tsai CC, Hong YJ, Lee RJ, Cheng NC, Yu J (2019) Enhancement of human adipose-derived stem cell spheroid differentiation in an in situ enzyme-crosslinked gelatin hydrogel. J Mater Chem B 7:1064 1075

Volponi AA, Sharpe PT (2013) The tooth - a treasure chest of stem cells. Br Dent J 215:353-358
Watt FM, Hogan BLM (2000) Out of Eden: stem cells and their niches. Science 287:1427-1430

Yamada M, Hori A, Sugaya S, Yajima Y, Utoh R, Yamato M, Seki M (2015) Cell-sized condensed collagen microparticles for preparing microengineered composite spheroids of primary hepatocytes. Lab Chip 15:3941-3951

Yang XT, Ma Y, Guo WH, Yang B, Tian WD (2019) Stem cells from human exfoliated deciduous teeth as an alternative cell source in bioroot regeneration. Theranostics 9:2694-2711 